\title{
Avaliação sorológica para deteç̧ão de anticorpos anti-Leishmania em cães e gatos no bairro de Santa Rita de Cássia, Município de Barra Mansa, Estado do Rio de Janeiro
}

\author{
Serological evaluation for detection of anti-Leishmania antibodies in \\ dogs and cats in the district of Santa Rita de Cássia, \\ municipality of Barra Mansa, State of Rio de Janeiro
}

\author{
Fabiano Borges Figueiredo ${ }^{1}$, Isabel Cristina Fábregas Bonna ${ }^{2}$, Lílian Dias Nascimento ${ }^{3}$, \\ Tatiana da Costa ${ }^{2}$, Cibele Baptista ${ }^{3}$, Tânia Maria Valente Pacheco', \\ Maria Regina Reis Amendoeira ${ }^{2}$ e Maria de Fátima Madeira ${ }^{3}$
}

\begin{abstract}
RESUMO
A partir de um caso canino de leishmaniose tegumentar americana na localidade de Santa Rita de Cássia, município de Barra Mansa, Rio de Janeiro, foi realizado um inquérito sorológico em 177 cães e 43 gatos. Na avaliação das amostras de soros caninos, 10\% foram positivos na reação de imunofluorescência indireta e 10,7\% no ensaio imunoenzimático. Entre as amostras de soros felinos testados, nenhum animal foi positivo na reação de imunofluorescência indireta e apenas um (2,4\%) felino apresentou reação positiva ao ensaio imunoenzimático. A detecção de Leishmania braziliensis, autóctone em Barra Mansa, faz um alerta para a instalação de um possível foco de leishmaniose tegumentar americana nessa região.
\end{abstract}

Palavras-chaves: Leishmaniose tegumentar americana. Diagnóstico. Sorologia. Cães e gatos. Barra Mansa.

\begin{abstract}
From a canine case of american cutaneous leishmaniasis in the locality of Santa Rita de Cássia, municipality of Barra Mansa, Rio de Janeiro, a serological survey was performed on 177 dogs and 43 cats. Evaluation of the canine serum samples showed that $10 \%$ had a positive reaction in the indirect immunofluorescence test and $\mathbf{1 0 . 7 \%}$ in the enzyme-linked immunosorbent assay. Among the feline serum samples tested, none of the animals had a positive reaction in the indirect immunofluorescence test and only one (2.4\%) showed a positive reaction in the enzyme-linked immunosorbent assay. The detection of an autochthonous case of Leishmania braziliensis in Barra Mansa gives warning that a focus of american cutaneous leishmaniasis is possibly becoming established in this region.
\end{abstract}

Key-words: American cutaneous leishmaniasis. Diagnosis. Serology. Dogs and cats. Barra Mansa.

As leishmanioses são zoonoses que acometem o homem e outras espécies de mamíferos silvestres e domésticos, de forma crônica com diversas manifestações clínicas. São causadas por protozoários do gênero Leishmania cuja transmissão ocorre através da picada de insetos vetores da subfamília Phlebotominae ${ }^{816}$.

A leishmaniose tegumentar americana (LTA) constitui um grave problema de saúde pública podendo ocasionar deformidades destrutivas e por vezes incapacitantes. No Brasil, apresenta ampla distribuição geográfica, sendo que o número de

1. Laboratório de Pesquisa Clínica em Dermatozoonoses em animais domésticos, Instituto de Pesquisa Clínica Evandro Chagas, Fundação Oswaldo Cruz, Rio de Janeiro, RJ. 2. Laboratório de Toxoplasmose, Instituto Oswaldo Cruz, Fundação Oswaldo Cruz, Rio de Janeiro, RJ. 3. Laboratório de Vigilância em Leishmanioses, Instituto de Pesquisa Clínica Evandro Chagas, Fundação Oswaldo Cruz, Rio de Janeiro, RJ.

Endereço para correspondência: Dr. Fabiano Borges Figueiredo. Laboratório de Pesquisa Clínica em Dermatozoonoses em Animais Domésticos/IPEC/FIOCRUZ. Av. Brasil 4365, 21045-900 Rio de Janeiro, RJ.

Tel: $55213865-9536$

e-mail: fabiano.figueiredo@ipec.fiocruz.br

Recebido para publicação em 17/12/2007

Aceito em 05/03/2009 casos vem aumentando progressivamente com uma média anual de 35 mil indivíduos infectados nos últimos $20 \operatorname{anos}^{122}$.

Leishmania (Viannia) braziliensis constitui um dos agentes etiológicos da LTA, cujo ciclo em ambiente urbano apresenta um perfil de transmissão no intra e no peridomicílio, condicionado à adaptação de algumas espécies de flebotomíneos ao meio ambiente da periferia das cidades, atingido também mulheres e crianças $^{691625}$. Neste contexto, é importante ressaltar a presença de hospedeiros domésticos como cães, felinos e eqüinos, criando a possibilidade, ainda não comprovada, desses animais atuarem como reservatórios e amplificadores de ciclos instalados no peridomicílio $^{10} 16$.

No Estado do Rio de Janeiro, a ocorrência da LTA é assinalada em diferentes municípios desde o início do século $X X^{9}$. Embora seja difícil comprovar a autoctonia dos casos nessa época, diversos outros surtos foram também descritos, denotando a expansão da LTA para outras regiões do estado ${ }^{2192331}$. 0 processo migratório aliado ao tipo de ocupação do solo, provocando situações de desordem ambiental, foi por diversas vezes associado ao aparecimento de novos casos de LTA no Rio de Janeiro ${ }^{13}{ }^{16}$. 
Durante um estudo no bairro de Santa Rita de Cássia, Barra Mansa (RJ) foi encontrado um cão naturalmente infectado por Leishmania sp., motivando este trabalho, através da busca ativa de possíveis outros casos em cães e gatos da região.

\section{MATERIAL E MÉTODOS}

O bairro de Santa Rita de Cássia está situado no município de Barra Mansa, região rural do Estado do Rio de Janeiro, cuja base da economia é a horticultura. A região se localiza na latitude de $22^{\circ} 29^{\prime \prime} 34^{\prime}, 46 \mathrm{~S}$ e longitude de 4409" $56^{\prime}, 30 \mathrm{~W}$, próximo à Serra das Araras e faz limite com o município de Volta Redonda (Figura 1).

Segundo a Secretaria Municipal de Saúde de Barra Mansa (SMS/BM) a população humana é de 1300 habitantes, com uma parte flutuante formada por pessoas que chegam à região para prestar serviços.

Entre os meses de julho e dezembro de 2005 foi realizada busca ativa de cães e gatos no bairro periférico de Santa Rita de Cássia, Barra Mansa, RJ. Durante as visitas domiciliares, os responsáveis pelos animais foram esclarecidos sobre o estudo e autorizaram a coleta de espécimes clínicos dos animais, assim como a utilização dos dados da pesquisa, através de um termo de consentimento. Este estudo foi aprovado pela Comissão de Ética no Uso de Animais da Fundação Oswaldo Cruz (CEUA/FIOCRUZ, P-0280/06). Como não havia informação prévia oficial sobre 0 número da população animal foi realizado um censo, com visitas em todas as residências da região.

Os animais foram contidos fisicamente para a coleta de sangue e exame clínico. Nos cães de grande porte foi puncionada a veia cefálica e em felinos e cães de médio e pequeno porte a veia de escolha foi a jugular. De cada animal foram coletados $5 \mathrm{~mL}$ de sangue e acondicionados em tubos estéreis (BD-Vacutainer) para posterior realização dos testes sorológicos. Após estes procedimentos, todos os animais foram submetidos ao exame clínico e dermatológico, buscando evidenciar a presença de lesões cutâneas e mucosas sugestivas de LTA.

0 teste de imunofluorescência indireta (IFI) para a pesquisa de anticorpos caninos anti-Leishmania foi realizada segundo a descrição do kit de IFI para o diagnóstico da leishmaniose visceral canina (Bio-Manguinhos/FIOCRUZ). Considerou-se positivas as amostras que apresentaram fluorescência em diluições iguais ou superiores a 1:40. Para o teste imunoenzimático (ELISA), utilizouse a pesquisa de imunoglobulinas da classe G (IgG) específicas para antígenos obtidos de formas promastigotas de Leishmania (Viannia) braziliensis (MHOM/BR/75/M2903). Os soros foram diluídos a 1:40 em duplicata e o ponto de corte (cut-off) foi estabelecido pela média das leituras dos controles negativos mais duas vezes o desvio padrão dos mesmos. Valores até $20 \%$ do cut-off foram considerados indeterminados e a positividade baseou-se em leituras superiores a esse valor.

$\mathrm{Na}$ avaliação sorológica felina, formas promastigotas de Leishmania (Viannia) braziliensis foram utilizadas como antígeno tanto para a IFI quanto para o ELISA. Na IFI, os soros foram diluídos a partir de 1:40 e o conjugado (Goat anti-cat IgG Fc fragment FITC, A20-117F, Bethyl Laboratory Inc., Montogomery, TX) diluído a 1:50 em salina tamponada com fosfatos (PBS) com 1:25 de Azul de Evans. Títulos iguais ou superiores a 1:40 foram considerados positivos. Para o ELISA, placas de poliestireno de 96 poços (Nunc Maxisorp, Nalgene Nunc International, Rochester, NY) foram sensibilizadas com 0 antígeno total parcialmente solúvel de Leishmania (Viannia) braziliensis. As amostras de soro foram diluídas a 1:40 e o conjugado (Goat anti-cat IgG Fc HRP) (A20-117P, Bethyl Laboratory Inc., Montogomery, TX) a 1:40.000. A determinação da positividade foi semelhante ao obtido no mesmo teste para os cães. No soro dos felinos foi realizado também o teste de ELISA rápido para detecção de antígenos do vírus da leucemia felina (FeLV) e anticorpos anti $o$ vírus da imunodeficiência felina (FIV) (SNAP Combo FeLVAg/

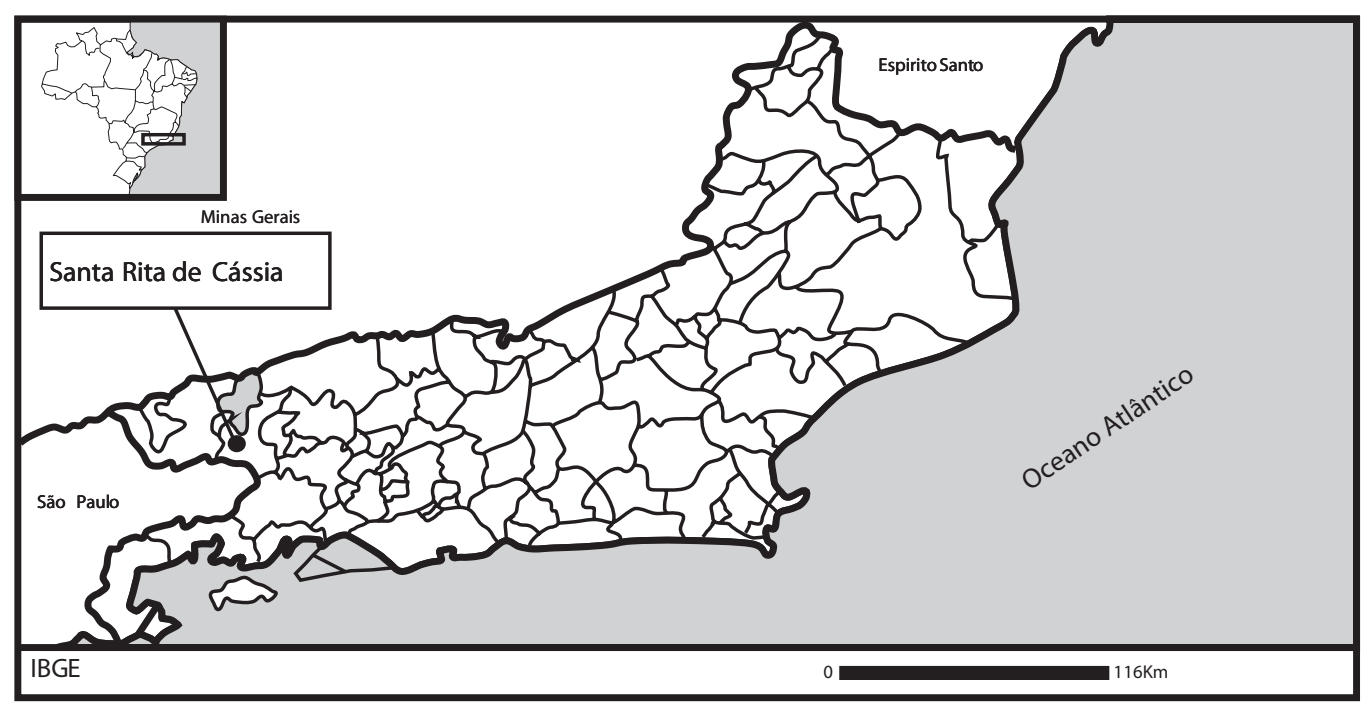

FIGURA 1

Mapa do Estado do Rio de Janeiro, assinalando a localidade de Santa Rita de Cássia no município de Barra Mansa.

Fonte: Adaptado de IBGE (http://mapas.ibge.gov.br/divisao/viewer.htm). 
FIVAb, IDEXX, Wesbrook, Mass). A técnica foi realizada de acordo com as instruções do fabricante.

No cão que apresentava lesão sugestiva, foi realizada biópsia visando o isolamento parasitário. Para este procedimento, o animal foi sedado por via intramuscular com acepromazina $(0,1-0,2 \mathrm{mg} / \mathrm{kg})$ e quetamina $(10 \mathrm{mg} / \mathrm{kg})$ e anestesiado no local da biópsia com lidocaína $2 \%$. Fragmentos obtidos do bordo da lesão foram conservados em solução fisiológica contendo 1.200UI de penicilina; 1.000 ug de estreptomicina e 100ug de 5' fluorocitosina por mililitro e após 24 horas foram semeados em meio de cultura bifásico NNN (Novy, MacNeal, Nicolle)/Schneider suplementado com $10 \%$ de soro fetal bovino. Os tubos foram incubados a temperatura de $26-28^{\circ} \mathrm{C}$ e examinados semanalmente. Os isolados obtidos foram caracterizados pela eletroforese de enzimas utilizando protocolos já definidos?.

\section{RESULTADOS}

No período do estudo, foram avaliados 177 cães (68 machos e 109 fêmeas) e 43 gatos (26 machos e 17 fêmeas). Dos soros caninos testados pela técnica de IFI, $10 \%$ apresentaram reatividade sorológica com os seguintes títulos: 1:40 (5,6\%); 1:80 (3,4\%); $1: 160(0,6 \%)$ e $1: 320(0,6 \%)$ e pelo teste de ELISA, $10,7 \%$ foram sororreatores e 5,6\% apresentaram reações indeterminadas. Entre os felinos avaliados, nenhum animal foi considerado reator à IFI; entretanto, ao ELISA, 2,4\% apresentaram reatividade e 4,8\% mostraram resultados indeterminados. No exame sorológico, para detecção dos vírus da leucemia felina e da imunodeficiência felina, todos os animais apresentaram resultados negativos.

Dos 220 animais avaliados dermatologicamente, apenas um cão apresentou uma lesão ulcerada na região da bolsa escrotal (Figura 2), cujo exame parasitológico possibilitou 0 isolamento de formas promastigotas em cultura, identificadas posteriormente, por isoenzimas como Leishmania (Viannia) braziliensis (Figura 3).

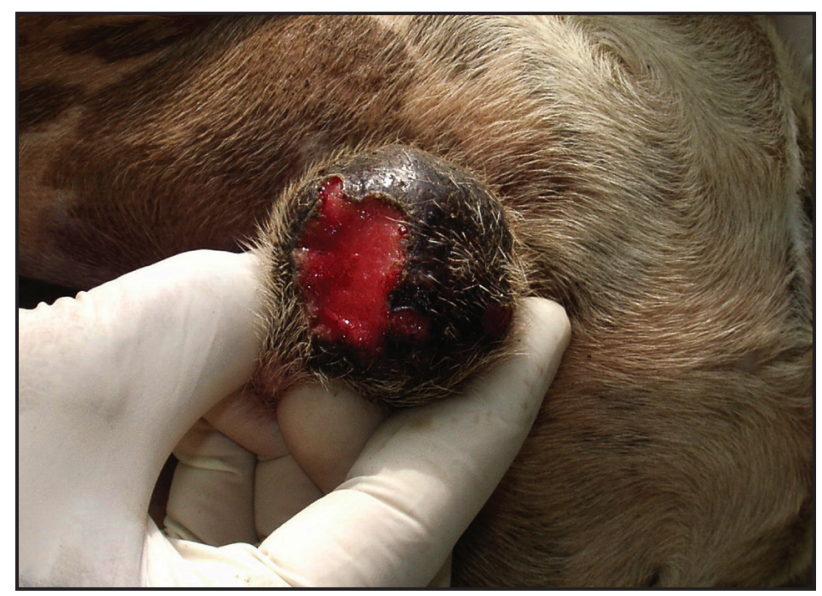

FIGURA 2

Lesão em bolsa escrotal de um canino infectado por Leishmania (Viannia) braziliensis.

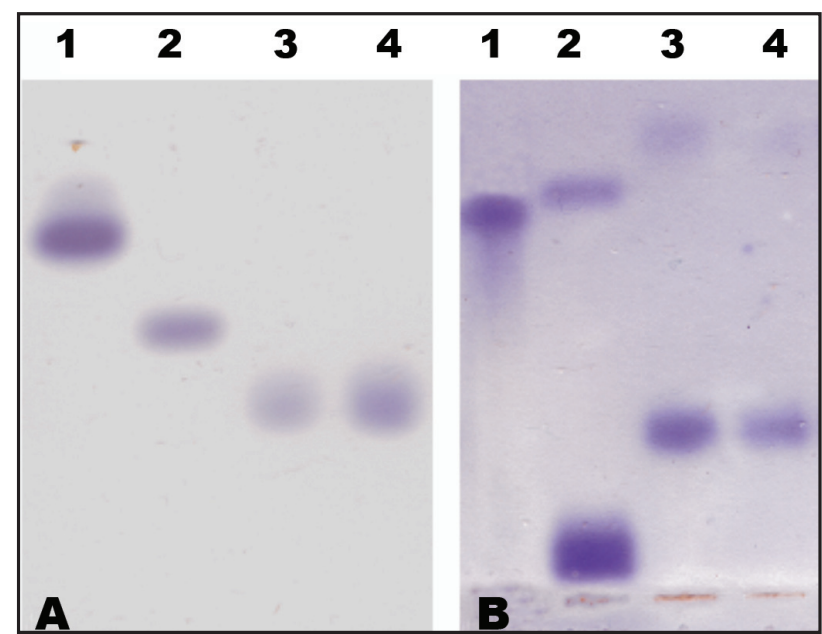

FIGURA 3

Perfil eletroforético apresentado pela enzima 6-phospho-glucomutase (A) e nucleotidase (B). 1: Leishmania (Leishmania) amazonensis (IFLA/BR/67/PH8); 2: Leishmania (Leishmania) chagasi (MHOM/BR/74/PP75); 3: Leishmania (Viannia) braziliensis (MHOM/BR/75/M2903); 4: Amostra do cão do estudo.

\section{DISCUSSÃO}

0 exame sorológico através das técnicas de IFI e ELISA já é consolidado em inquéritos caninos, para a detecção da leishmaniose visceral apresentando altas taxas de sensibilidade e especificidade ${ }^{2124}$. Entretanto, os resultados dessas metodologias relacionados a forma cutânea da doença são discordantes.

Eminfecções humanas por Leishmania (Viannia) braziliensis é descrito que a resposta imune é predominantemente celular, determinando assim fraca resposta humoral e baixa quantidade de anticorpos séricos detectáveis pelos métodos tradicionais ${ }^{1}$.

Uchoa cols ${ }^{33}$ sugerem que mesmo em cães as técnicas sorológicas devam ser utilizadas associadas a outros parâmetros ou então para definições de diagnósticos diferenciais da LTA. Já em outro estudo realizado em cães de área endêmica no Rio de Janeiro, mostrou que a detecção de anticorpos específicos, através dos testes sorológicos como a IFI e ELISA, apresentou maior sensibilidade e especificidade quando comparados ao exame clínico e a demonstração do parasita no exame direto ${ }^{3}$. Associado a este achado Silveira cols ${ }^{32}$ e Zanzarini cols ${ }^{35}$ relataram que em comparação com a técnica de IFI, o esfregaço por aposição não constitui um processo ideal de diagnóstico para LTA, devido ao baixo número de parasitas presentes nas lesões desses animais. Em nosso estudo, embora 220 animais tenham sido cuidadosamente examinados, o parasita foi constatado em apenas um cão, que apresentou reatividade para ambos os testes sorológicos com o título de 1:320 na IFI.

A prevalência sorológica canina encontrada neste estudo foi semelhante à encontrada em outras áreas endêmicas de LTA $^{1128}$. Outro resultado importante foi o encontro de cães sorologicamente positivos sem a presença de lesões o que reforça o relato de Marzochi e Barbosa-Santos em 1988, os quais sugerem a existência de animais infectados por Leishmania sem a presença deste sinal clínico ${ }^{15}$. Estes resultados fazem um alerta para a possível instalação de um surto na região. 
Com relação aos valores das titulações encontradas na IFI, 94,4\% (n=178) dos cães apresentaram títulos iguais ou inferiores a 1:160, resultado esse que se assemelha ao descrito na literatura ${ }^{3} 1732$

Na comparação entre as técnicas de IFI e ELISA, os resultados mostraram percentuais similares, diferenciando dos achados de Uchoa cols ${ }^{33}$ onde $24,5 \%$ dos animais $(n=261)$ apresentaram reatividade no ELISA e apenas $0,4 \%$ pela IFI. Barbosa cols $^{3}$ mostraram uma prevalência na IFI de 3,2\% e no ELISA de 10,2\% em 215 cães estudados.

A busca por leishmaniose felina na região estudada justificase pelos relatos da infecção natural desse animal em diferentes regiões do Brasil ${ }^{18262930}$ e do mundo ${ }^{4527}$, o que sugere um possível envolvimento na epidemiologia das leishmanioses.

Dos 43 gatos examinados, embora nenhum tenha apresentado lesões cutâneas, a prevalência sorológica foi semelhante aos achados de Poli cols ${ }^{27}$, onde foi encontrado uma positividade de $0,9 \%$, em 110 felinos avaliados pela técnica de IFI, e aos resultados de Michael $\operatorname{cols}^{20}$, que utilizando o teste de hemaglutinação indireta, relataram $3,7 \%$ de positividade em felinos do Egito, sendo inferior às prevalências encontradas por Vita $\operatorname{cols}^{34}(16,3 \%) \mathrm{e}$ Martin-Sanchez cols ${ }^{14}(28,3 \%)$.

Apesar da região estudada não ser considerada endêmica para LTA, o inquérito sorológico realizado nos cães mostrou um percentual elevado de animais positivos. 0 isolamento de Leishmania em meio de cultura e posterior identificação etiológica confirmam a circulação da Leishmania (Viannia) braziliensis no bairro de Santa Rita de Cássia, município de Barra Mansa, RJ sendo necessários estudos mais detalhados principalmente sobre a fauna flebotomínica local e sobre a exposição da população humana frente à infecção deste parasita.

\section{AGRADECIMENTOS}

Agradecemos à população da comunidade de Santa Rita de Cássia pela colaboração, paciência e apoio à equipe de pesquisadores de campo.

\section{REFERÊNCIAS}

1. Ajdary S, Alimohammadian MH, Eslami MB, Kemp K, Kharazmi A, Coutinho SD. Comparison of the immune profile of nonhealing cutaneous leishmaniasis patients with those with active lesions and those rK39: a cloned antigen of Leishmania chagas $i$ that predicts active visceral leishmaniasis. Journal of Infectious Diseases 173:758-761, 1996

2. Araújo Filho NA, Coura JR. Leishmaniose tegumentar americana na Ilha Grande, Rio de Janeiro. IV. Reservatórios domésticos. Revista da Sociedade Brasileira de Medicina Tropical 15:82-103, 1978.

3. Barbosa GM, Marzochi MC, Massard CL, Lima GP, Confort EM. Epidemiological aspects of canine american tegumentary leishmaniasis in the municipality of Paraty, State of Rio de Janeiro, Brazil. Cadernos de Saúde Pública 15:641-646, 1999.

4. Barnes JC, Stanley 0, Craig TM. Diffuse cutaneous leishmaniasis in a cat. Journal of the American Veterinary Medical Association 202:416-418, 1993.

5. Bonfante-Garrido R, Urdaneta I, Urdaneta R, Alvarado J. Natural infection of cats with Leishmania in Barquisimeto, Venezuela. Transactions of the Royal Society of Tropical Medicine and Hygiene 85:53, 1991.
6. Cerqueira AGC, Vasconcelos, A. A leishmaniose nesta capital. Boletim Sanitário Publicações Científicas do Departamento Nacional de Saúde Pública 1:35-47, 1922.

7. Cupolillo E, Grimaldi Jr G, Momen H. A general classification of new world Leishmania using numeral zymotaxomomy. The American Journal of Tropical Medicine and Hygiene 50:296-311, 1994.

8. Deane LM, Deane MP. Visceral Leishmaniasis in Brazil: Geographical Distribution and Transmission. Revista do Instituto de Medicina Tropical de São Paulo 4:198$212,1962$.

9. D’Utra-e-Silva 0. Sobre a leishmaniose tegumentar e seu tratamento. Memórias do Instituto Oswaldo Cruz 7:213-248, 1915.

10. Ferreira WA, MayrinkW, Mares-Guia ML, Tavares CA. Detection and characterization of Leishmania antigens from an American cutaneous leishmaniasis vaccine for diagnosis of visceral leishmaniasis. Diagnostic Microbiology of Infectious Diseases 45:35-43, 2003.

11. Follador I, Araujo C, Cardoso MA, Tavares-Neto J, Barral A, Miranda JC, Bittencourt A, Carvalho EM. Outbreak of American cutaneous leishmaniasis in Canoa, Santo Amaro, Bahia, Brazil. Revista da Sociedade Brasileira de Medicina Tropical 32:497-503, 1999.

12. Gontijo B, de Carvalho ML. American cutaneous leishmaniasis. Revista da Sociedade Brasileira de Medicina Tropical 36:71-80, 2003.

13. Kawa H, Sabroza PC. Spatial distribution of tegumentary leishmaniasis in the city of Rio de Janeiro. Cadernos de Saúde Pública 18:853-865, 2002.

14. Martin-Sanchez J, Acedo C, Munoz-Perez M, Pesson B, Marchal 0, Morillas-Marquez F. Infection by Leishmania infantum in cats: Epidemiological study in Spain. Veterinary Parasitololy 145:267-273, 2007.

15. Marzochi MC, Barbosa-Santos EG. Evaluation of a skin test on the canine mucocutaneous leishmaniasis diagnosis. Memórias do Instituto Oswaldo Cruz 83:391-392, 1988.

16. Marzochi MCA, Marzochi KBF. Tegumentary and visceral leishmaniasis in Brazil: emerging anthropozoonosis and possibilities for their control. Cadernos de Saúde Pública 10:359-375, 1994

17. Maywald PG, Machado MI, Costa-Cruz JM, Gonçalves-Pires M. Canine cutaneous and visceral leishmaniasis and Chagas' disease from counties in the Triangulo Mineiro and Alto Paranaiba regions, Minas Gerais State, Brazil. Cadernos de Saúde Pública 12:321-328, 1996.

18. Mello GB. Verificação da infecção natural do gato (Felix domesticus) por um protozoário do genero Leishmania. Brasil Médico 54:180, 1940.

19. Menezes JA, Reis VLL, Vasconcellos JA. Pequeno surto de leishmaniose tegumentar americana em Macuco (Cordeiro - RJ). Revista da Sociedade Brasileira de Medicina Tropical 8:1134-1151, 1974.

20. Michael SA, Morsy TA, El-Seoud SF, Saleh MS. Leishmaniasis antibodies in stray cats in Ismailiya Governorate, Egypt. Journal of the Egyptian Society of Parasitology 12:283-286, 1982.

21. Ministério da Saúde. Manual de Vigilância e Controle da Leishmaniose Visceral. Série A. Normas e Manuais Técnicos: $1^{\text {a }}$ edição. Brasília, 2006.

22. Ministério da Saúde. Manual de Vigilância da Leishmaniose Tegumentar Americana. Série A. Normas e Manuais Técnicos: $2^{a}$ edição. Brasília, 2007.

23. Nery-Guimarães F. Estudo de um foco de Leishmaniose muco-cutânea na Baixada Fluminense (estado do Rio de Janeiro). Memórias do Instituto Oswaldo Cruz 53:1-11, 1955

24. Oliveira LS, Julião FS, Souza VMM, Freitas DS, Souza BMPS, Paule BJA, Aguiar PHP, Melo SMB, Franke CR. A utilização da imunofluorescência indireta no diagnóstico de rotina da leishmaniose visceral canina e suas implicações no controle da doença. Ciência Animal Brasileira 6:41-47, 2005.

25. Passos VM, Falcão AL, Marzochi MC, Gontijo CM, Dias ES, Barbosa-Santos EG, Guerra HL, Katz N. Epidemiological aspects of American cutaneous leishmaniasis in a periurban area of the metropolitan region of Belo Horizonte, Minas Gerais, Brazil. Memórias do Instituto Oswaldo Cruz 88:103-110, 1993.

26. Passos VM, Lasmar EB, Gontijo CM, Fernandes 0, Degrave W. Natural infection of a domestic cat (Felis domesticus) with Leishmania (Viannia) in the metropolitan region of Belo Horizonte, State of Minas Gerais, Brazil. Memórias do Instituto Oswaldo Cruz 91:19-20, 1996. 
27. Poli A, Abramo F, Barsotti P, Leva S, Gramiccia M, Ludovisi A, Mancianti F. Feline leishmaniosis due to Leishmania infantum in Italy. Veterinary Parasitology 106:181-191, 2002.

28. Santos GP, Sanavria A, Marzochi MC, Santos EG, Silva VL, Pacheco RS, Mouta-Confort E, Espíndola CB, Souza MB, Ponte CS, Conceição NF, Andrade MV. Prevalence of canine infection from endemic areas of American cutaneous leishmaniasis in Paracambi District, Rio de Janeiro State, between 1992 and 1993. Revista da Sociedade Brasileira de Medicina Tropical 38:161-166, 2005.

29. Savani ES, Oliveira Camargo MC, Carvalho MR, Zampieri RA, Santos MG, D'Auria SR, Shaw JJ, Floeter-Winter LM. The first record in the Americas of an autochthonous case of Leishmania (Leishmania) infantum chagasi in a domestic cat (Felix catus) from Cotia County, Sao Paulo State, Brazil. Veterinary Parasitology 120:229-233, 2004.

30. Schubach TM, Figueiredo FB, Pereira SA, Madeira MF, Santos IB, Andrade MV, Cuzzi T, Marzochi MC, Schubach A. American cutaneous leishmaniasis in two cats from Rio de Janeiro, Brazil: First report of natural infection with Leishmania (Viannia) braziliensis. Transactions of the Royal Society of Tropical Medicine and Hygiene 98:165-167, 2004.
31. Serra CM, Leal CA, Figueiredo FB, Schubach TM, Duarte R, Uchoa CM, Silva RM Madeira MF. Canine tegumentary leishmaniasis in Morada das Águias (Serra da Tiririca), Maricá, Rio de Janeiro, Brazil. Cadernos de Saúde Pública 19:1877$1880,2003$.

32. Silveira TG, Teodoro U, Lonardoni MV, Toledo MJ, Bertolini DA, Arraes SM, Filho DV. Serologic survey of dogs in an endemic area of tegumentary leishmaniasis in Parana State, southern Brazil. Cadernos de Saúde Pública 12:89-93, 1996.

33. Uchoa CM, Serra CMB, Duarte R, Magalhães CM, Silva RM, Theophilo F, Figliuolo LP, Horta FT, Madeira MF. Serological and epidemiological features of canine American tegumentary Leishmaniasis from Marica, Rio de Janeiro, Brazil. Revista da Sociedade Brasileira de Medicina Tropical 34:563-568, 2001.

34. Vita S, Santori D, Aguzzi I, Petrotta E, Luciani A. Feline leishmaniasis and ehrlichiosis: serological investigation in Abruzzo region. Veterinary Research Communications 29:319-321, 2005.

35. Zanzarini PD, Santos DR, Santos AR, Oliveira O, Poiani LP, Lonardoni MV Teodoro U, Silveira TGV. Canine American cutaneous leishmaniasis in municipalities of northern Parana State, Brazil. Cadernos de Saúde Pública 21:1957-1961, 2005. 